\title{
Cardia Orifice
}

National Cancer Institute

\section{Source}

National Cancer Institute. Cardia Orifice. NCI Thesaurus. Code C32262.

The region distal to the $z$-line of the gastroesophageal junctions, the area where the esophagus attaches to the stomach. 